\title{
An improved numerical method to compute neutron/gamma deexcitation cascades starting from a high spin state
}

\author{
D. Regnier ${ }^{\mathrm{a}, \mathrm{b}}$, O. Litaize ${ }^{\mathrm{a}}$, O. Serot ${ }^{\mathrm{a}}$ \\ ${ }^{a}$ CEA, DEN, DER, SPRC, Cadarache, F-13108 Saint Paul lez Durance, France \\ ${ }^{b}$ Nuclear and Chemical Science Division, Lawrence Livermore National Laboratory, Livermore, CA 94551, USA
}

\begin{abstract}
Numerous nuclear processes involve the deexcitation of a compound nucleus through the emission of several neutrons, gamma-rays and/or conversion electrons. The characteristics of such a deexcitation are commonly derived from a total statistical framework often called "Hauser-Feshbach" method. In this work, we highlight a numerical limitation of this kind of method in the case of the deexcitation of a high spin initial state. To circumvent this issue, an improved technique called the Fluctuating Structure Properties (FSP) method is presented. Two FSP algorithms are derived and benchmarked on the calculation of the total radiative width for a thermal neutron capture on ${ }^{238} \mathrm{U}$. We compare the standard method with these FSP algorithms for the prediction of particle multiplicities in the deexcitation of a high spin level of ${ }^{143} \mathrm{Ba}$. The gamma multiplicity turns out to be very sensitive to the numerical method. The bias between the two techniques can reach $1.5 \gamma /$ cascade. Finally, the uncertainty of these calculations coming from the lack of knowledge on nuclear structure is estimated via the FSP method.
\end{abstract}

Keywords: Hauser-Feshbach ; statistical model ; deexcitation ; cascade ; FIFRELIN ;

\section{Introduction}

Numerous nuclear reactions involve the formation of an excited compound nucleus. These nuclei may then lose their energy through the emission of several neutrons, gamma-rays and possibly conversion electrons. Given the initial bounded or virtual level $\left(E J^{\pi}\right)_{i}$ of such a nucleus, a standard problem resides in the determination of a few average observables characterizing the deexcitation process. Such calculations have a broad field of applications including namely the prediction of:

- The gamma multiplicities and spectra in capture reactions at low incident neutron energy [1, 2]. Beyond the prediction of gamma observables, these calculations are also required to infer indirectly the spin of the resonances [3, 4], or the branching ratio of some isomeric states [5].

- The prompt neutron and gamma emissions from fission fragments $[6,7,8]$, as well as their spin distribution [9, $10,11,12,13,14]$.

- The spin distribution of the compound nuclei involved in surrogate reaction studies [15].

These phenomena have been widely calculated using one statistical technique. For every nuclei involved in the deexcitation cascade, experimental data provides the decay structure at low energies. The high part of the excitation energy scale is discretized in small intervals (bins). Inside these intervals,

${ }^{*}$ Corresponding author.

E-mail address: olivier.litaize@cea.fr all the structure properties are considered constant equal to an averaged value. The probability of a transition between two bins can then be determined. Based on these probabilities, a deterministic or Monte Carlo technique is applied to compute an average observable over all possible deexcitation cascades. This statistical technique is often referred as a Hauser-Feshbach method [16]. However, in this particular context, no entry channel is involved in the calculation. Therefore this method does not take into account any correlations between channels. In this paper, we refer to this method as the Average Structure Properties (ASP) method. Indeed, the structure properties of the nuclei involved are averaged over small energy bins.

The ASP method has been successfully used in many applications. However, we discovered that a numerical issue rises in the case of a specific application: the calculation of the neutron/gamma deexcitation from nuclei with high spin values. To circumvent this drawback, we propose an other method that will be referred as the Fluctuating Structure Properties (FSP) method. This method consists in a generalization of the work of Becvar [17] on gamma/electron cascades calculation. We will show that this FSP method yields a more physical behavior when applied to nuclei with high spin values. Moreover, it also estimates the uncertainties coming from our lack of knowledge on the nuclear structure.

The present article is organized as follows. First of all, the section 2 presents briefly the standard ASP deexcitation algorithm. We emphasize the numerical issue of the ASP method by calculating the neutron emission probability $\mathrm{P}_{n}$ for a range of initial excited levels in a ${ }^{143} \mathrm{Ba}$ compound nucleus. This last isotope was chosen because it is a highly yielded primary fragment in the spontaneous fission of ${ }^{252} \mathrm{Cf}$ (sf) [18]. The section 3 
is devoted to the new FSP method. After a complete description of the principle, two algorithms are derived. The neutron emission probability $\mathrm{P}_{n}$ obtained with these algorithms for ${ }^{143} \mathrm{Ba}$ are highlighted in perspective of the results coming from the ASP method. Finally, the section 4 compares the three algorithms (one for ASP and two for FSP). We test the consistency of our implementation by calculating the total radiative width of an excited state of ${ }^{238} \mathrm{U}$ (a standard in nuclear data evaluations). Then we discuss the neutron and gamma multiplicities computed with the three algorithms for a ${ }^{143} \mathrm{Ba}$ deexcitation process.

\section{The Average Structure Properties (ASP) method}

\subsection{Description and notations}

Let us consider a nucleus in an initial level $(E, J, \pi)_{i}$, where $E$ stands for the excitation energy, and $J^{\pi}$ are the quantum numbers associated with the total angular momentum and the parity. We assume that this initial level is not stable and that the nucleus will deexcite through the emission of several neutrons, gamma-rays and/or conversion electrons. Our goal is to calculate the average of an observable Q (e.g. a particle multiplicity) over all the possible cascades:

$$
Q_{\mathrm{ASP}}=\sum_{c} Q(c) p(c)
$$

where $Q(c)$ is the value of $Q$ given the cascade $c$ and $p(c)$ is the probability that the cascade $c$ happens. Given the probabilities $p(c)$, both a deterministic or a Monte Carlo numerical method can compute $Q_{\text {ASP. }}$ In this work, a large amount of cascade events are sampled according to the distribution $p(c)$. Each event increments an estimator of $Q_{\text {ASP. }}$.

The probabilities $p(c)$ are drastically dependent on the structure properties of every nuclei involved in the deexcitation process. An exact computation of these probabilities would require:

1. The exact level scheme of every nuclei involved. This level scheme has to be known up to an energy $E_{\text {top }}(A)$. For the initial nucleus $E_{t o p}(A)$ is the initial excitation energy. After each neutron emission, it decreases by the corresponding binding energy:

$$
E_{\text {top }}(A-1)=E_{\text {top }}(A)-S_{n}(A)
$$

2. The partial widths of every possible transitions from any initial to any final level. These levels may not belong to the same nucleus.

Whereas these properties are not known in such details for masses $A>50$, some statistical information is available (e.g. the level density). This incomplete knowledge can be used to approximate the probabilities $p(c)$.

In the ASP method, the level scheme of any nucleus is divided into two energy regions separated by an energy denoted $E_{\text {cut-off }}$. In the low energy range $\left(E<E_{\text {cut-off }}\right)$, the level scheme consists in the discrete levels provided by an experimental or evaluated data base. In the high energy range
$\left(E>E_{c u t-o f f}\right)$, the excitation energy scale is divided into bins of an arbitrary width $\mathrm{dE}$. In that region, the nucleus is no more characterized by its excited level but only by its energy bin and its $J^{\pi}$ value. In order to clarify the discussion, it is convenient to define a level set noted $\left[E J^{\pi}\right]$. It corresponds either to the set of $J^{\pi}$ levels with an energy in $[E-d E / 2 ; E+d E / 2]$, either to a discrete level $(E, J, \pi)$. We also note $N\left(\left[E J^{\pi}\right]\right)$ the number of levels contained in the set $\left[E J^{\pi}\right]$. If the level set actually corresponds to a discrete level, this quantity is equal to one. The ASP method estimates the number of levels $N\left(\left[E J^{\pi}\right]\right)$ as an average value given by:

$$
N\left(\left[E J^{\pi}\right]\right)= \begin{cases}\rho(E, J, \pi) \cdot \mathrm{dE} & \text { for an energy bin } \\ 1 & \text { for one discrete level }\end{cases}
$$

where $\rho(E, J, \pi)$ designates the level density of the nucleus.

The average partial width for a transition $\left[E J^{\pi}\right]_{i}$ to $\left[E J^{\pi}\right]_{f}$ with the emission of a particle $p$ of energy $E_{p}$ and characterized by a set $\alpha$ of quantum numbers is expressed as:

$$
\begin{aligned}
\Gamma_{p}\left(\left[E J^{\pi}\right]_{i} \rightarrow\left[E J^{\pi}\right]_{f}, \alpha\right) & = \\
& \left\langle\sum_{n_{f} \in\left[E J^{\pi}\right]_{f}} \Gamma_{p}\left(n_{i} \rightarrow n_{f}, \alpha\right)\right\rangle_{n_{i} \in\left[E J^{\pi}\right]_{i}}
\end{aligned}
$$

It is obtained by summing the level to level partial widths over final levels and averaging over initial levels. Such a partial width can also be written:

$$
\begin{aligned}
\Gamma_{p}\left(\left[E J^{\pi}\right]_{i} \rightarrow\right. & {\left.\left[E J^{\pi}\right]_{f}, \alpha\right)=} \\
& \bar{\Gamma}_{p}\left(E_{p}, \alpha\right) \cdot \delta\left(\alpha, J_{i}^{\pi}, J_{f}^{\pi}\right) \cdot N\left(\left[E J^{\pi}\right]_{f}\right)
\end{aligned}
$$

The Kronecker's symbol $\delta$ accounts for the selection rules and is evaluated to 1 if the transition is possible and 0 otherwise. The factor $\bar{\Gamma}_{p}\left(E_{p}, \alpha\right)$ is the average width of the possible transitions. In this model, it is assumed to be independent of $J \pi_{i}$ and $J \pi_{f}$. This quantity can be derived from several advanced analytic models [19, 20, 21] or as a result of a microscopic calculation [22].

Once all the widths $\Gamma_{p}\left(\left[E J^{\pi}\right]_{i} \rightarrow\left[E J^{\pi}\right]_{f}, \alpha\right)$ are determined, the probability of a specific emission from an initial set $\left[E J^{\pi}\right]_{i}$ is given by the ratio:

$$
\begin{aligned}
p\left(\left[E J^{\pi}\right]_{i}\right. & \left.\rightarrow\left[E J^{\pi}\right]_{f}, p, \alpha\right)= \\
& \frac{\Gamma_{p}\left(\left[E J^{\pi}\right]_{i} \rightarrow\left[E J^{\pi}\right]_{f}, \alpha\right)}{\Gamma^{t o t}}
\end{aligned}
$$

where $\Gamma^{t o t}$ is the sum of the partial widths over all possible deexcitations from $\left[E J^{\pi}\right]_{i}$. The probability $p(c)$ of a deexcitation cascade $c$ can then be written as a product of probabilities calculated with Eq. 5. Eventually, injecting the probability of every possible cascade in Eq. 1 provides the targeted observable $Q_{\text {ASP. }}$

\subsection{Implementation details}

A Monte Carlo resolution of Eq. 1 based on the ASP method has been implemented in the code FIFRELIN, dedicated to the 
simulation of the fission fragment deexcitation [23, 24]. The description of the structure of the nuclei relies on the RIPL3 [25] database. In particular, both the energy cutoff $\left(E_{\text {cut }-o f f}\right)$ and the discrete levels below this energy are directly taken from RIPL-3. Above $E_{c u t-o f f}$, the energy scale is divided into bins of $10 \mathrm{keV}$ width. We checked that calculations performed with $5 \mathrm{keV}$ bins lead to the same conclusions.

The total level density used in Eq. 2 is a Composite Gilbert Cameron Model [26]. For each nucleus, three constraints determine the parametrization of the level density:

- The level density is continuous at the matching energy, i.e. between the Constant Temperature part (low energies) and the Fermi Gaz model part (high energies).

- The derivative of the level density is continuous at the matching energy.

- The number of levels in an energy range $\left[E_{\min } ; E_{\text {max }}\right]$ matches the RIPL-3 database. The energies $E_{\min }$ and $E_{\max }$ are themselves provided by RIPL-3.

The total level density reads:

$$
\rho(E, J, \pi)=\rho_{t o t}(E) \cdot p_{J}^{\pi}\left(J^{\pi}, E\right)
$$

The two parity values are assumed equiprobable. The spin parity distribution is defined as:

$$
p_{J}^{\pi}\left(E, J^{\pi}\right) \propto \frac{1}{2} \frac{2 J+1}{2 \sigma^{2}(E)} \cdot \exp \left(-\frac{(J+1 / 2)^{2}}{2 \sigma^{2}(E)}\right)
$$

The energy dependency of the spin cutoff $\sigma(E)$ follows the prescription given in the section VI.A.2.c of RIPL-3 [25].

The average gamma partial width for a transition of type $X=$ $E, M$ and multipolarity $L$ is computed via a gamma strength function $f_{X L}\left(\epsilon_{\gamma}\right)$ :

$$
\bar{\Gamma}_{\gamma}\left(\epsilon_{\gamma}, X L\right)=\frac{f_{X L}\left(\epsilon_{\gamma}\right) \epsilon_{\gamma}^{2 L+1}}{\rho_{i}\left(E_{i}, J_{i}, \pi_{i}\right)},
$$

For the E1 electric dipole transitions, we use the enhanced generalized Lorentzian model of strength function as defined in RIPL-3. For M1 magnetic dipole transitions, a standard Lorentzian model is adopted. Its parameters are coming from the RIPL-3 systematics. The strengths of higher multipolarities is considered proportional to $f_{E 1}$. The Weisskopf estimations of gamma widths (cf. [27]) provide us the proportionality factor. Below $E_{\text {cut-off }}$ the experimental intensities of the possible transitions may be available in RIPL-3. In that case, the deexcitation probabilities are computed directly from these data. If a conversion coefficient is also available, the code takes into account the possible emission of an electron.

If the neutron emission channel is open, its average partial width for a given neutron orbital momentum $l$ and total angular momentum $j$ is determined via the formula:

$$
\bar{\Gamma}_{n}\left(\epsilon_{n}, l, j\right)=\frac{T_{l, j}\left(\epsilon_{n}\right)}{2 \pi \rho_{i}\left(E_{i}, J_{i}, \pi_{i}\right)}
$$

The neutron transmission coefficients $T_{l, j}\left(\epsilon_{n}\right)$ are provided by an optical model calculation. These coefficients are first evaluated and stored for a wide range of nuclei and for a set of neutron energies using TALYS-1.4 [28]. The neutron energy grid consists in 1000 points ranging from $1 \mathrm{eV}$ to $20 \mathrm{MeV}$. The evaluated energies are distributed in such a way that the maximal error due to a linear interpolation in that mesh is of the order of a few percents. During the Monte Carlo calculation, the transmission coefficients are linearly interpolated between the stored coefficients.

\subsection{Calculation of the neutron emission probability from ${ }^{143} \mathrm{Ba}$}

This part aims to emphasize a limitation of the ASP method in the case of the neutron/gamma deexcitation from a high spin initial state. The ASP method is applied to a ${ }^{143} \mathrm{Ba}$ with excitation energies ranging in the interval $\left[S_{n} ; S_{n}+3.5 \mathrm{MeV}\right]$. This calculation is repeated for various initial spin-parity values. The structures of ${ }^{143} \mathrm{Ba}$ and ${ }^{142} \mathrm{Ba}$ coming from RIPL-3 are shown in Fig. 1. This type of neutron-rich nucleus is typically encountered when computing the prompt particles emission from fission. With such an energy/spin of the initial state, one could assume that only neutron, gamma and conversion electron emissions are possible. Our goal is to determine the total probability of a neutron emission from the initial state.

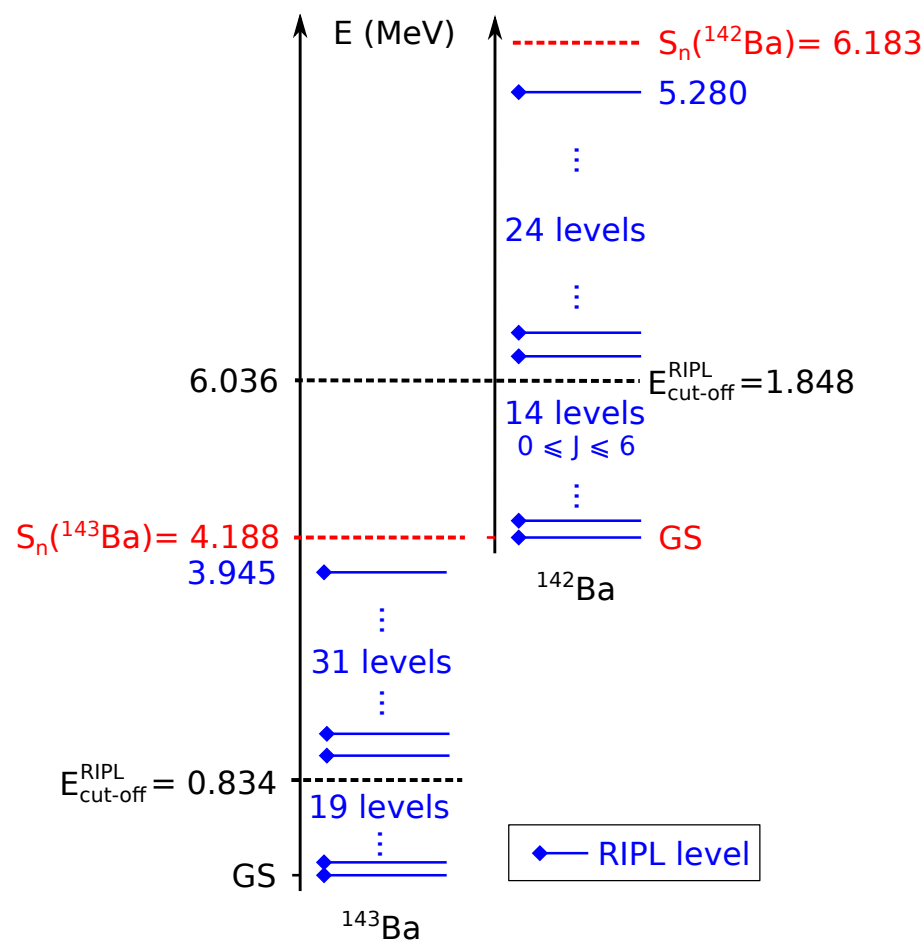

Figure 1: Nuclear structure of ${ }^{142} \mathrm{Ba}$ and ${ }^{143} \mathrm{Ba}$ taken from RIPL-3. All energies are given in $\mathrm{MeV}$.

The probability of a neutron emission from the initial level set reads:

$$
\mathrm{P}_{n}\left(\left[E J^{\pi}\right]_{i}\right)=\Gamma_{n}^{\mathrm{tot}} /\left(\Gamma_{\gamma}^{\mathrm{tot}}+\Gamma_{n}^{\mathrm{tot}}\right)
$$

The total gamma and neutron widths from the initial level set $\left(\Gamma_{\gamma}^{\mathrm{tot}}, \Gamma_{n}^{\mathrm{tot}}\right)$ are obtained by summing the respective partial widths 
over all the possible transitions:

$$
\Gamma_{p}^{\text {tot }}\left(\left[E J^{\pi}\right]_{i}\right)=\sum_{\left[E J^{\pi}\right]_{f}, \alpha} \Gamma_{p}\left(\left[E J^{\pi}\right]_{i} \rightarrow\left[E J^{\pi}\right]_{f}, \alpha\right)
$$

Figure 2 shows the calculated probability $\mathrm{P}_{n}\left(\left[E J^{\pi}\right]_{i}\right)$ as a function of $E_{i}$. It also emphasizes the evolution of these functions with the initial spin $J_{i}$. The initial state has an imposed positive parity.

Given one spin for the initial level, the behavior of $\mathrm{P}_{n}$ as a function of the excitation energy presents three different zones.

- At low energy above $S_{n}$, the gamma emission prevails. The neutron emission is energetically possible. However, the difference between the initial spin and the spin of the reachable levels imposes that an emitted neutron would have a high orbital momentum. The partial widths of such neutron emissions are much weaker than the total gamma width. In the extreme cases where $J_{i}<5 / 2$, this zone is nonexistent because the neutron emission probability to the fundamental state is already stronger than the radiative width.

- From a given energy above $S_{n}$, the neutron emission probability skyrockets. At the transition energy, it becomes possible to populate a final level by the emission of a neutron with a low orbital momentum. The partial width associated to this particular transition is of the same order of magnitude than the total gamma width. As soon as the initial excitation energy grows up, that particular width increases faster than the total gamma width. In addition, other similar neutron channels can open up. These two effects induce a sharp increase of the probability to emit a neutron up to 1 . The transition takes place in only a few hundreds of $\mathrm{keV}$. The opening of a new neutron channel during the transition phase results in a break of the slope of $\mathrm{P}_{n}$. Such an effect can be observed for the initial spins $J_{i}=7.5$ and 8.5 at $S_{n}+1.47 \mathrm{MeV}$. It corresponds to the opening of the 7th level of the ${ }^{142} \mathrm{Ba}$ with a spin parity of $6+$.

- For energies above the transition phase, the neutron emission prevails.

Let us now consider the evolution of the functions $\mathrm{P}_{n}\left(E_{i}\right)$ with the initial spin $J_{i}$. At low energy and spin, pairs of consecutive $J_{i}$ values give similar neutron emission probability. The even-even structure of ${ }^{142} \mathrm{Ba}$ associated with the selection rules for the neutron emission explain this behavior for the decay from positive parity states. For example, the neutron transmission related to a transition from a $1.5^{+}$state to the ground state of ${ }^{142} \mathrm{Ba}\left(0^{+}\right)$is given by $T_{l=2, j=1.5}$. On the other hand, a transition $2.5^{+} \rightarrow 0^{+}$is characterized by $T_{l=2, j=2.5}$. These two coefficients are close enough to yield the same $\mathrm{P}_{n}\left(E_{i}\right)$ functions for $J_{i}=1.5$ and 2.5. As the spin and excitation energy increase, openings of different neutron channels as well as disparity between $T_{l, j=l+1 / 2}$ and $T_{l, j=l-1 / 2}$ split such pairs of neutron emission probability functions.

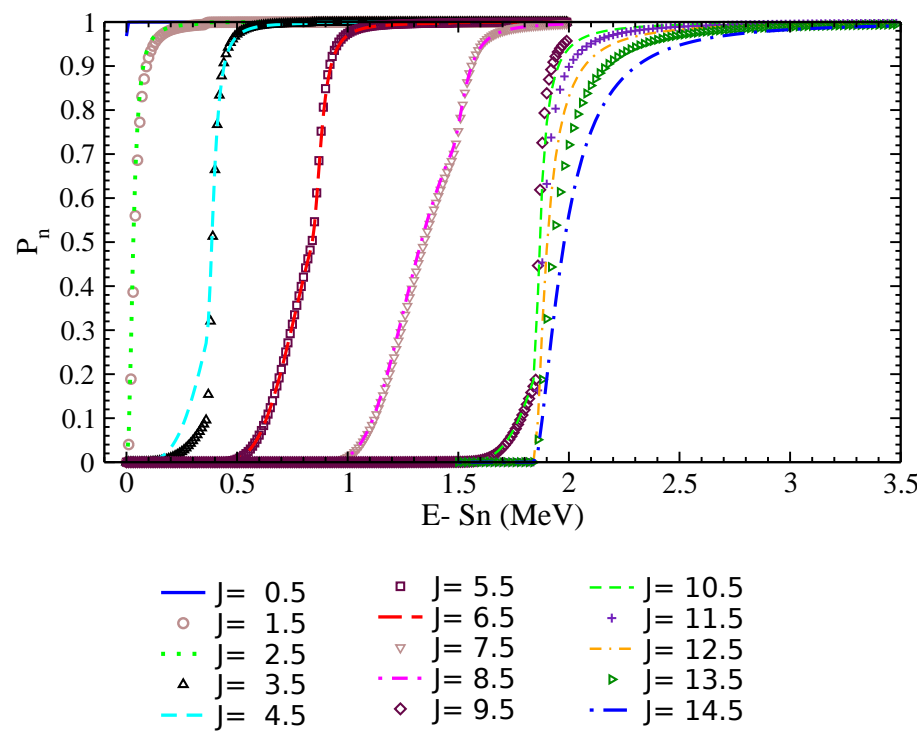

Figure 2: Probability of a neutron emission from an excited level set $\left[E J^{\pi}\right]_{i}$ of ${ }^{143} \mathrm{Ba}$ as a function of the excitation energy and for different initial spins. The initial parity is positive. These calculations are based on the ASP method.

For $J_{i} \leq 8.5$, the energy at which the transition between gamma and neutron emission happens increases with $J_{i}$. As extensively developed in $[29,30,31]$, this behavior is directly linked to the Yrast line of the ${ }^{142} \mathrm{Ba}$. As $J_{i}$ increases, the energies of the reachable levels in ${ }^{142} \mathrm{Ba}$ raise. Therefore, the transition zone is expected to drift continuously toward the high energies. However, for all $J_{i}>8.5$, the ASP method predicts a transition happening constantly at $1.85 \mathrm{MeV}$ above the neutron separation energy. This nonphysical behavior can actually be linked with the approximations assumed on the structure of ${ }^{142} \mathrm{Ba}$. Below $S_{n}+1.85 \mathrm{MeV}$, the level scheme of ${ }^{142} \mathrm{Ba}$ is only composed of discrete levels. The Yrast line is correctly taken into account. It shifts the transition energy as $J_{i}$ increases. Above $S_{n}+1.85 \mathrm{MeV}$, the level scheme of ${ }^{142} \mathrm{Ba}$ is discretized into bins. In this energy domain, the ASP method favors drastically the neutron emission probability from the states $J_{i}>8.5$.

\section{Fluctuating Structure Properties (FSP) method}

In the work of Becvar [17], a numerical technique going beyond some assumptions of the ASP method is exposed. This algorithm is implemented in the code DICEBOX and treats only gamma/conversion electrons deexcitation cascades. We generalize this work to the case of neutron/gamma/conversion electrons deexcitation cascades. The aim is to overcome the numerical issue highlighted in section 2.3.

\subsection{Global overview}

In his work, Becvar introduces the notion of a nuclear realization. It corresponds both to the complete level scheme (E, $J^{\pi}$ values for every level) and the associated gamma partial widths (for every possible transition) for one nucleus. We generalize that notion for the case where several nuclei are involved in the decay chain. 
- Let a nuclear realization $\omega$ denote the level schemes and the associated partial widths of every nucleus involved in the decay chain.

- Let $\Omega$ be the set of nuclear realizations $\omega$ that respect for every of these nuclei the following conditions:

1. For $E<E_{\text {cut-off }}$, the level scheme and the decay probabilities are matching the structure data coming from a database (e.g. RIPL-3).

2. For $E>E_{\text {cut-off }}$, the level scheme is consistent with a theoretical level density $\rho(E, J, \pi)$.

3. For $E>E_{c u t-o f f}$, the partial widths of a given transition energy respect a theoretical distribution. According to the work of Porter et al.[32], this distribution will be defined as a $\chi^{2}$ distribution centered on a theoretical average width.

- Let $p(\omega)$ be a probability defined on $\Omega$.

It is then assumed that one particular nuclear realization $\omega^{\phi}$ of $\Omega$ corresponds to the actual structure properties of the nuclei involved in the deexcitation process.

Given one nuclear realization $\omega$, the probability $p(c \mid \omega)$ that a deexcitation cascade $c$ happens is determined. If $Q$ is one characteristic of the deexcitation, it is possible to compute the average $Q(\omega)$ over all cascades as:

$$
\begin{gathered}
Q(\omega)=\sum_{c} Q(c, \omega) \cdot p(c \mid \omega) \\
\sum_{c} p(c \mid \omega)=1
\end{gathered}
$$

As a probability has been defined on $\Omega$, the quantity $Q(\omega)$ can also be seen as a random variable. The two first momenta of its distribution represented in Fig. 3 are denoted as:

$$
\begin{aligned}
\mu(Q) & =\sum_{\omega \in \Omega} Q(\omega) p(\omega), \\
\sigma^{2}(Q) & =\sum_{\omega \in \Omega}[Q(\omega)-\mu(Q)]^{2} p(\omega)
\end{aligned}
$$

Following this formalism, the physical quantity that is sought

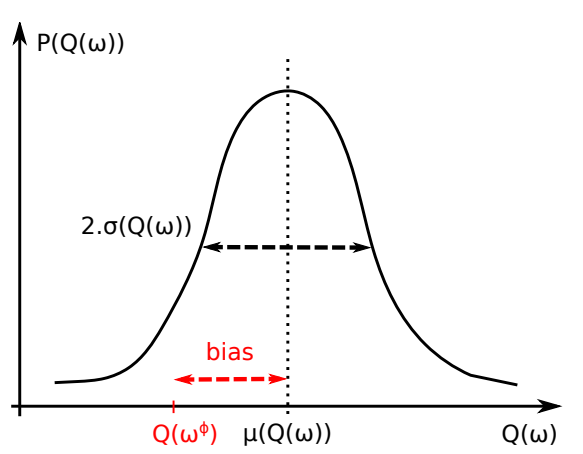

Figure 3: Interpretation of the momenta in term of physical observable

is $Q\left(\omega^{\phi}\right)$. The exact $\omega^{\phi}$ is not known, only the set $\Omega$ is known at this step. However, the observable $Q$ may not strongly depend on the variations of $\omega$ in $\Omega$. In this case one could assume that the physical quantity sought is not so far from the average of the $Q$ distribution:

$$
Q_{\mathrm{FSP}}=\mu(Q) \simeq Q\left(\omega^{\phi}\right) \pm \sigma(Q)
$$

The standard deviation $\sigma(Q)$ can be interpreted as the uncertainty of this result due to the lack of knowledge on the structure properties. If $\sigma(Q)$ is small enough compared to a given error criteria, then the approximation 15 is justified.

\subsection{Monte Carlo estimation of the momenta $\mu(Q)$ and $\sigma^{2}(Q)$}

Numerically, the two first momenta of the $Q(\omega)$ distribution can be evaluated in a Monte Carlo framework. The calculation scheme of the FSP method is illustrated in Fig. 4. It consists in $N_{\Omega}$ sampling of a nuclear realization for which the quantity $Q(\omega)$ is estimated through the sampling of $N_{c}$ cascades. Based on such a sample, an observable can actually be estimated by the mean of the three unbiased estimators $\hat{\mu}(Q), \hat{\sigma^{2}}(\hat{\mu}(Q))$ and $\hat{\sigma^{2}}(Q)$. These estimators provide an evaluation of $\mu(Q)$, its statistical uncertainty and $\sigma^{2}(Q)$ respectively. They are computed using a method similar to the one given in Ref. [17]. All of them are unbiased Monte Carlo estimators. In terms of numerical computation, the storage of a complete nuclear realization is not possible. To circumvent this problem we used a technique already described in Ref. [17] consisting in storing only the random seeds necessary to build the nuclear realization. Each time that the level schemes and partial widths of a few nuclei are needed in the cascade sampling process, they are built again from the stored random seeds.

\subsection{Sampling of the nuclear realizations}

Two different methods have been used to sample nuclear realizations.

\subsubsection{Bin Sampling (FSP-b)}

In this method, the level scheme is divided into two parts separated by $E_{\text {cut-off }}$. The low energy part contains the discrete levels coming from a data base. The high energy part is split in energy bins of $10 \mathrm{keV}$ width. Once again, we checked that calculations using $5 \mathrm{keV}$ bins lead to the same conclusions. During the generation of a nuclear realization $\omega$, the number of levels $N_{\omega}\left(\left[E J^{\pi}\right]\right)$ in a level set above $E_{c u t-o f f}$ is sampled between the two closest integers from $\rho(E, J, \pi) \mathrm{dE}$. This sampling is classically done such as the average value is $\rho(E, J, \pi) \mathrm{dE}$. For every possible transition, a fluctuation factor is sampled to take into account the Porter Thomas fluctuations [32] of the partial widths. We assume that the level to level partial widths are $\chi^{2}$ distributed with one degree of freedom. The fluctuation factor $y_{f, \omega}(i \rightarrow f, \alpha)$ of the partial width associated to a transition $\left[E J^{\pi}\right]_{i} \rightarrow\left[E J^{\pi}\right]_{f}$ is then sampled in a $\chi^{2}$ distribution with $N_{\omega}\left(\left[E J^{\pi}\right]_{i}\right) \times N_{\omega}\left(\left[E J^{\pi}\right]_{f}\right)$ degrees of freedom. 


\begin{abstract}
$\mathrm{N}_{\Omega}$ : $\quad$ Number of nuclear realizations $\mathrm{n}_{\Omega} \in\left[1, \mathrm{~N}_{\Omega}\right]$ : Current nuclear realization
\end{abstract}

$\begin{array}{ll}\mathrm{N}_{\mathrm{C}}: & \text { Number of cascades } \\ \mathrm{n}_{\mathrm{C}} \in\left[1, \mathrm{~N}_{\mathrm{C}}\right]: & \text { per nuclear realization } \\ \text { Current cascade }\end{array}$

Current cascade

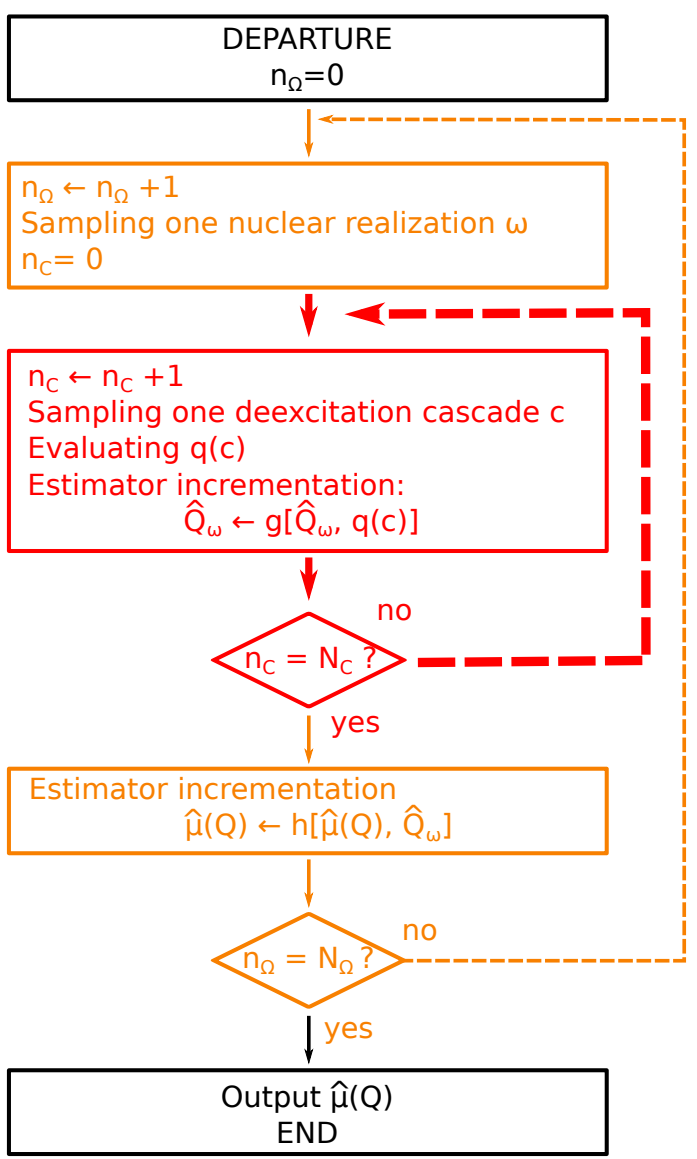

Figure 4: Simplified scheme of the algorithm for the evaluation of an estimator $\hat{\mu}(Q)$

If no experimental data is available for a transition, the partial widths are calculated as:

$$
\begin{aligned}
& \Gamma_{p}\left(\left[E J^{\pi}\right]_{i} \rightarrow\left[E J^{\pi}\right]_{f}, \alpha\right)= \\
& \bar{\Gamma}_{p}\left(E_{p}, \alpha\right) \cdot y_{f, \omega}(i \rightarrow f, \alpha) \times \\
& \delta\left(\alpha, J \pi_{i}, J \pi_{f}\right) \cdot N_{\omega}\left(\left[E J^{\pi}\right]_{f}\right)
\end{aligned}
$$

This algorithm does not rely anymore on an averaged non integer number of levels in each energy bin. In addition, this method attempts to take into account the Porter Thomas fluctuations of the partial widths.

\subsubsection{Level Sampling (FSP-l)}

This last algorithm aims to get ride of the energy bin approximation. We first considered a discrete level treatment of the full level schemes of the nuclei. However, this brute force method turned out to be too much time and memory consuming. In the following algorithm, the level scheme is divided into three parts (cf. Fig. 5):

- from $E^{*}=0$ to $E_{\text {cut-off }}$ : the level scheme is only filled with discrete levels from a database,

- from $E_{\text {cut-off }}$ to an energy $E_{\text {bin }}$ : available level data from the database are put into the level scheme. Then additional discrete levels are sampled to complete this energy region based on a theoretical level density.

- above $E_{b i n}$ : the level scheme is discretized in bins having their properties sampled as described in the FSP-b method.

From a computational point of view, only the second region differs from the previous methods. In this energy range, the few levels available in the structure database are collected and added in the scheme. The spin parity of these levels may be missing. If so, they are sampled from the distribution $P_{J}^{\pi}\left(J^{\pi}, E\right)$ defined in Eq. 7. This step populates the middle energy region with a few levels. However, the density of levels in this first scheme is lower than the theoretical density $\rho(E, J, \pi)$. A second step consists in completing the scheme by sampling and adding new levels. Before any new level creation, our implementation compares the cumulative number of levels in the scheme versus the cumulative inferred from $\rho(E, J, \pi)$. The addition of new levels stops when the two cumulatives differ only by a few units in the whole energy range.

The boundary $E_{\text {bin }}$ is determined as a compromise between computation performances and results accuracy. In this work, we chose this limit based on the criteria: $\rho_{\text {tot }}\left(E_{\text {bin }}\right)=$ $10^{5}$ levels/MeV. Increasing this value has shown to modify the results only within a percent. Once all the level schemes are built, the partial widths between their level sets are calculated using Eq. 16.

In this last algorithm the energy binning approximation is only applied in energy ranges where level density takes high values. Moreover, additional structure data coming from the database can easily be added in the deexcitation calculation.

\subsection{Calculation of the neutron emission probability from ${ }^{143} \mathrm{Ba}$}

We implemented the Monte Carlo resolution of Eq. 15 for the two variants of the nuclear realization sampling (FSP-b and FSP-1). This section presents the neutron emission probability from a ${ }^{143} \mathrm{Ba}$ deexcitation calculated with the FSP methods. We use the same level densities, gamma strengths, neutron transmissions and level database as in section 2.3 . Only the numerical method changes.

The Figure 6 highlights the results produced with both FSPb and FSP-1 algorithms. The results do not show anymore the numerical issue encountered with the ASP method (Fig. 2). The energy of the neutron/gamma transition is increasing with $J_{i}$ even for the highest initial spin. This behavior reproduces the expected effect of the ${ }^{142} \mathrm{Ba}$ Yrast line. It depends no more on the value of $E_{\text {cut }- \text { off }}$.

This striking difference between the ASP and FSP methods may a priori come from the change in the numerical treatment of the unknown nuclear properties, and/or the addition of the 

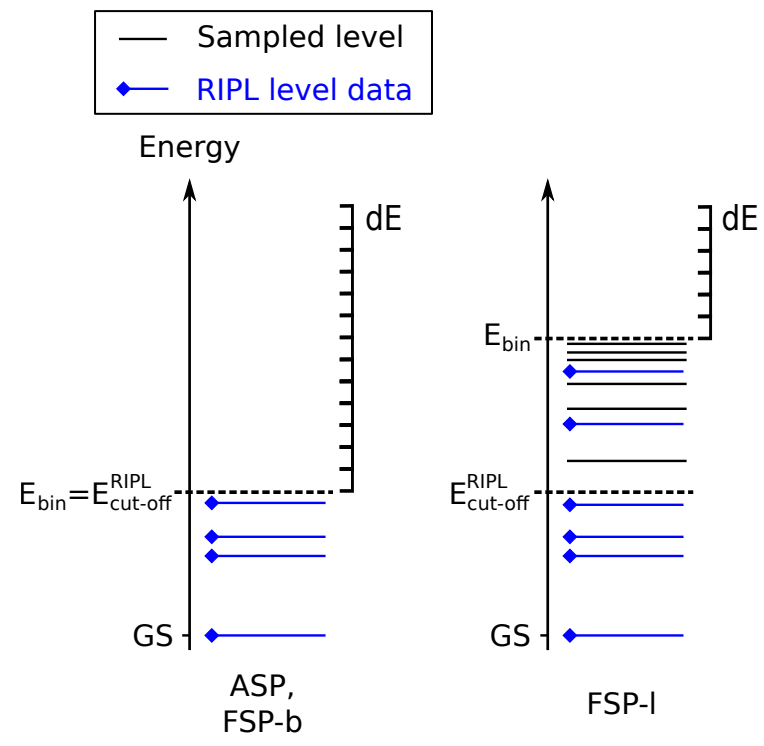

Figure 5: Level scheme treatment within the three algorithms emphasized in this paper

Porter-Thomas fluctuations in the model. The second hypothesis can actually be dismissed by performing a FSP-b calculation with no such fluctuations. Removing the Porter-Thomas fluctuations increases slightly the neutron emission probability. Figure 7, shows the difference between FSP calculations performed with and without fluctuations from an initial state $J_{i}=13.5$. The absolute difference $\Delta$ observed is not larger than 0.1 . Therefore the Porter-Thomas fluctuations are not responsible for the change in behavior under investigation.

To better understand the fundamental difference between the results obtained with the ASP and FSP methods, one should express their respective estimation of the neutron emission probability:

$$
\begin{aligned}
& P_{n, \mathrm{ASP}}=\frac{\Gamma_{n}^{\mathrm{tot}}(\bar{\omega})}{\Gamma_{n}^{\mathrm{tot}}(\bar{\omega})+\Gamma_{\gamma}^{\mathrm{tot}}(\bar{\omega})} \\
& P_{n, \mathrm{FSP}}=\left\langle\frac{\Gamma_{n}^{\mathrm{tot}}(\omega)}{\Gamma_{n}^{\mathrm{tot}}(\omega)+\Gamma_{\gamma}^{\mathrm{tot}}(\omega)}\right\rangle_{\omega \in \Omega}
\end{aligned}
$$

The FSP method computes an average of the final observable $\left(P_{n}\right)$ on the unknown structure properties. Conversely, the ASP method calculates the observable based on one "averaged nuclear realization" $\bar{\omega}$. Due to the non linear evolution of $P_{n}$ as a function of the widths, these two expressions can differ drastically. The ASP method misses here the correlations between the numerator and the denominator of Eq.17.

To better highlight the importance of such correlations, let us consider a typical scenario in the deexcitation of the ${ }^{143} \mathrm{Ba}$. Suppose that we are interested in the neutron emission probability starting from a high spin state $21 / 2^{+}$at an energy just above $S_{n}\left({ }^{143} \mathrm{Ba}\right)+E_{\text {cut-off }}\left({ }^{142} \mathrm{Ba}\right)=6.036 \mathrm{MeV}$. To simplify the discussion, we consider only the s wave neutron emissions that will populate the final level set $\left[E J^{\pi}\right]_{f}=\left[1.853 \mathrm{MeV} ; 10^{+}\right]$ of the ${ }^{142} \mathrm{Ba}$ scheme. In this picture, the deexcitation will either produce a gamma-ray, either a neutron with an energy close to
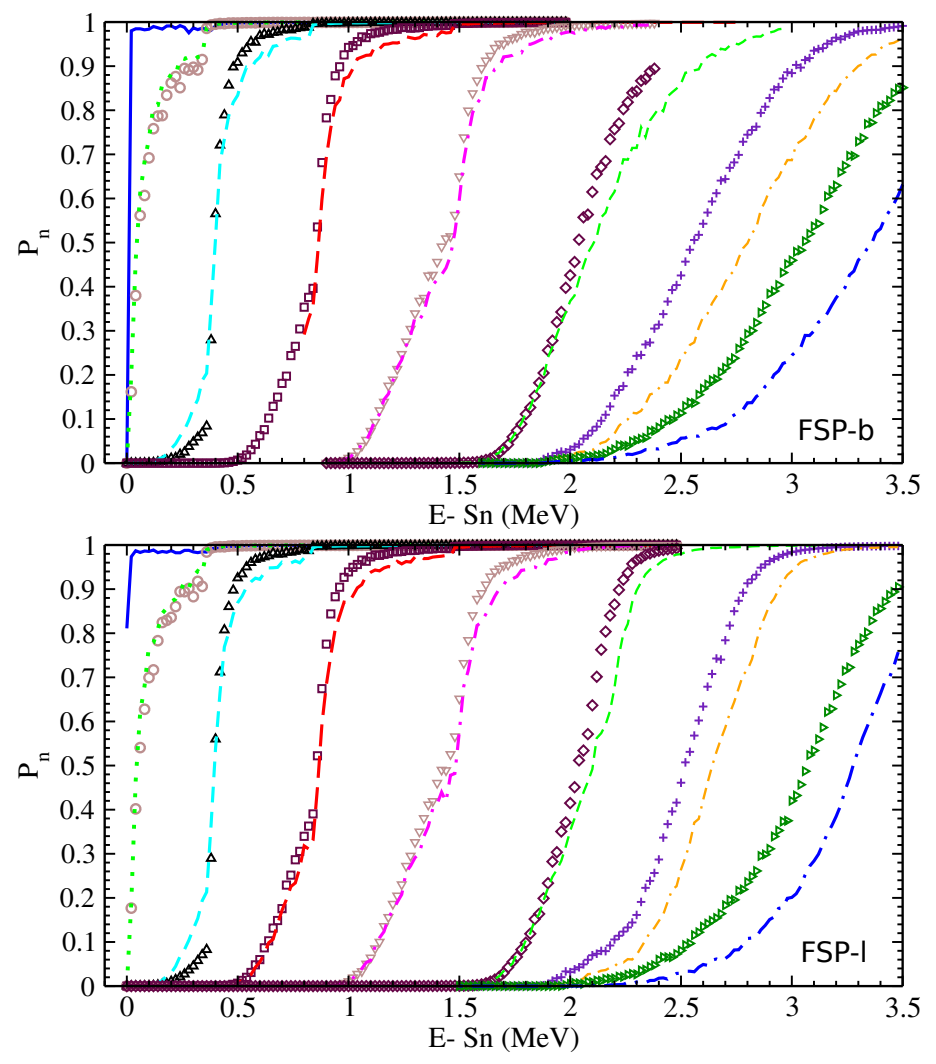

$\begin{array}{rrr}-J=0.5 & \square J=5.5 & --J=10.5 \\ \therefore J=1.5 & --J=6.5 & +J=11.5 \\ \because J=2.5 & \nabla J=7.5 & -\cdot J=12.5 \\ \Delta J=3.5 & -\cdot J=8.5 & \triangleright J=13.5 \\ --J=4.5 & \diamond J=9.5 & --J=14.5\end{array}$

Figure 6: Probability of a neutron emission from an excited level set $\left[E J^{\pi}\right]$ of a ${ }^{143} \mathrm{Ba}$. The probability is given as a function of the excitation energy and for different initial spins. The initial parity is positive. From the top to the bottom, the plots correspond to the results obtained with the FSP-b and the FSP-1 algorithms.

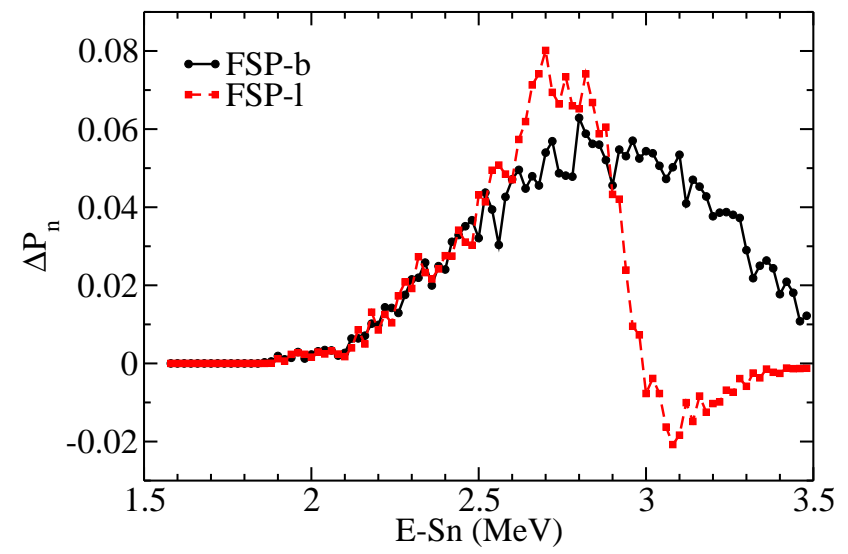

Figure 7: Effect of the Porter-Thomas fluctuations on the neutron emission probability from a $13.5^{+}$excited state of ${ }^{143} \mathrm{Ba}$. The symbol $\Delta$ denotes the difference ( $\left.P_{n \text {,without fluctuations }}-P_{n \text {,with fluctuations }}\right)$. This difference is calculated both with the FSP-b and FSP-1 methods. 
$5 \mathrm{keV}$. We note $\bar{\Gamma}_{n}$ the average neutron width (cf. Eq. 9) associated with the neutron transition. Let us now assume that the final level set has a probability $\alpha$ to contain one level and $(1-\alpha)$ to be empty. The probabilities for the neutron emission can be expressed as:

$$
\begin{aligned}
& P_{n, \mathrm{ASP}}=\frac{\alpha \bar{\Gamma}_{n}}{\Gamma_{\gamma}^{t o t}+\alpha \bar{\Gamma}_{n}} \\
& P_{n, \mathrm{FSP}}=\alpha \cdot \frac{\bar{\Gamma}_{n}}{\Gamma_{\gamma}^{t o t}+\bar{\Gamma}_{n}}
\end{aligned}
$$

The ratio of probability between the two methods is then given by:

$$
\frac{P_{n, \mathrm{ASP}}}{P_{n, \mathrm{FSP}}}=\frac{1+\bar{\Gamma}_{n} / \Gamma_{\gamma}^{t o t}}{1+\alpha \cdot \bar{\Gamma}_{n} / \Gamma_{\gamma}^{t o t}}
$$

In such a situation, we first notice that the ASP method will always overestimate the neutron emission probability. The amplitude of this bias is ruled by the ratio $\bar{\Gamma}_{n} / \Gamma_{\gamma}^{\text {tot }}$. Indeed, the deviation will be important as soon as the partial width of the neutron transition considered has at least the same order of magnitude than the total radiative width. If $\bar{\Gamma}_{n} / \Gamma_{\gamma}^{\text {tot }}$ is large enough, the ASP/FSP ratio is roughly inverse proportional to the probability $\alpha$. Note that the probability $\alpha$ is itself given by $\rho\left(E_{f}, J_{f}^{\pi}\right) \cdot d E$. In this example, the ratio $\bar{\Gamma}_{n} / \Gamma_{\gamma}^{\text {tot }}$ can be extracted from our implementation. It turns out to be roughly equal to 130 . Moreover, the level density in ${ }^{142}$ Ba yields a probability $\alpha \simeq 5 \cdot 10^{-3}$. These numerical values lead finally to probabilities:

$$
P_{n, \mathrm{FSP}} \simeq 5.10^{-3}, \quad P_{n, \mathrm{ASP}} \simeq 0.4
$$

The predictions from the two numerical methods differ by a factor 80 .

This example is by far too much simplified to provide any quantitative estimation. However, it gives us a clue on the key quantities and mechanisms which explain the difference between the FSP and ASP results. It seems that deexcitation cascades involving high spin values often present crucial transitions with both:

- a high ratio $\bar{\Gamma}_{n} / \Gamma_{\gamma}^{t o t}$,

- a low value for $\rho\left(E_{f}, J_{f}^{\pi}\right) \cdot d E$.

These conditions imply high fluctuations in the value of $\Gamma_{n}^{\text {tot }}(\omega)$. In contrast, the radiative width shows little variations with $\omega$. These two factors injected in Eq. 17 explain the differences observed between Fig. 2 and 6.

\section{Comparison of the ASP and FSP methods on several de- excitation characteristics}

\section{1. $n_{t h}+{ }^{238} U$ radiative width}

The total gamma width of a thermal neutron capture on a ${ }^{238} \mathrm{U}$ is calculated based on the three algorithms described in the previous sections. The incident neutron is assumed to be a pure s-wave so that the compound nucleus is necessary excited with a spin parity $\frac{1}{2}+$ and at the energy close to $S_{n}\left({ }^{239} U\right)=4.8$
$\mathrm{MeV}$. The level density, neutron transmission, gamma strengths and discrete levels used are the same as described in section 2.2. The energy $E_{\text {cut-off }}$ is $373 \mathrm{keV}$, and the bin treatment for FSP-1 begins at $E_{b i n}=3.34 \mathrm{MeV}$. The calculation of the total radiative width does not require the computation of the cascade probabilities. It only consists in summing the partial widths for all possible transitions from the initial state. The interest of such a calculation is that the ASP and the FSP methods should yield the same value for the total radiative width. Indeed, one can write the following equations:

$$
\begin{aligned}
& \Gamma_{\gamma, \text { FSP-b }}^{\mathrm{tot}} \\
& \quad=\left\langle\Gamma_{\gamma}^{\mathrm{tot}}(\omega)\right\rangle_{\omega \in \Omega} \\
& \quad=\sum_{f, \alpha}\left\langle\Gamma_{\gamma, \mathrm{FSP}-\mathrm{b}}\left(\left[E J^{\pi}\right]_{i} \rightarrow\left[E J^{\pi}\right]_{f}, \alpha\right)\right\rangle_{\omega \in \Omega} \\
& \quad=\sum_{f, \alpha} \Gamma_{\gamma, \mathrm{ASP}}\left(\left[E J^{\pi}\right]_{i} \rightarrow\left[E J^{\pi}\right]_{f}, \alpha\right) \\
& \quad=\Gamma_{\gamma, \mathrm{ASP}}^{\mathrm{tot}}
\end{aligned}
$$

A similar result can be obtained with FSP-1 as soon as no new level data from the structure library is injected above $E_{\text {cut-off }}$. We use this property to benchmark our implementations of the ASP and FSP methods.

The numerical results obtained are reported in Table 1 . In the case of the FSP methods, the radiative width is estimated by sampling 5000 nuclear realizations. The statistical uncertainty $(1 \sigma)$ due to the Monte Carlo estimation is reported. For the FSP-1 algorithm, no level data above $E_{\text {cut-off }}$ is used even if it is available in the structure library.

\begin{tabular}{lll}
\hline ASP & FSP-b & FSP-1 (no additional level) \\
\hline 17.69 & $17.71 \pm 0.01$ & $17.67 \pm 0.01$ \\
\hline
\end{tabular}

Table 1: Total gamma width of the thermal neutron capture on ${ }^{238} \mathrm{U}$ (in meV)

The order of magnitude of the radiative width is coherent with the 23.0 and $23.36 \mathrm{meV}$ values present in the JEFF-3.2 evaluation and the Atlas of neutron resonances [33] respectively. A different choice of photon strength function could typically explain such a $5 \mathrm{meV}$ difference. For instance, we obtained a radiative width of $23.62 \pm 0.04$ by using:

- the FSP-1 method with all the additional level data available in RIPL-3,

- a Composite Gilbert Cameron model for the level density,

- the HFB+QRPA tabulated values of the photon strength functions provided in RIPL-3.

As expected, the radiative widths presented in Tab. 1 for the different algorithms are equal within the statistical uncertainty. This result gives us confidence in our various implementations. In addition, we emphasize that the numerical issue highlighted in section 2.3 does not impact the calculation of a total radiative width.

\subsection{Particle multiplicities in the deexcitation of ${ }^{143} \mathrm{Ba}$}

\subsubsection{Reference comparison}

One of the main goal of the deexcitation codes is to predict the particle multiplicities and spectra. In this part, we compute 
the multiplicity of the neutrons and gamma-rays from a ${ }^{143} \mathrm{Ba}$ deexcitation cascade. The spin/parity of the initial state is set to $23 / 2+$. For such a high spin, the ASP method has an issue with the prediction of the total neutron emission probability. Given the spin/parity, we studied the evolution of the particles multiplicities as a function of the initial excitation energy $E \in[4,10]$ $\mathrm{MeV}$. This work is repeated with the three algorithms described previously. For the FSP-1 method, every available data from RIPL-3 is included. The ASP calculations are based on $2.10^{5}$ cascade events. The FSP results are obtained with 2000 cascades for each of the 400 nuclear realizations sampled. In all the presented results, the maximal uncertainty $(1 \sigma)$ coming from the Monte Carlo process is 0.02 and 0.06 for the neutron and gamma multiplicity respectively. Figure 8 presents the neutron and gamma multiplicities obtained.
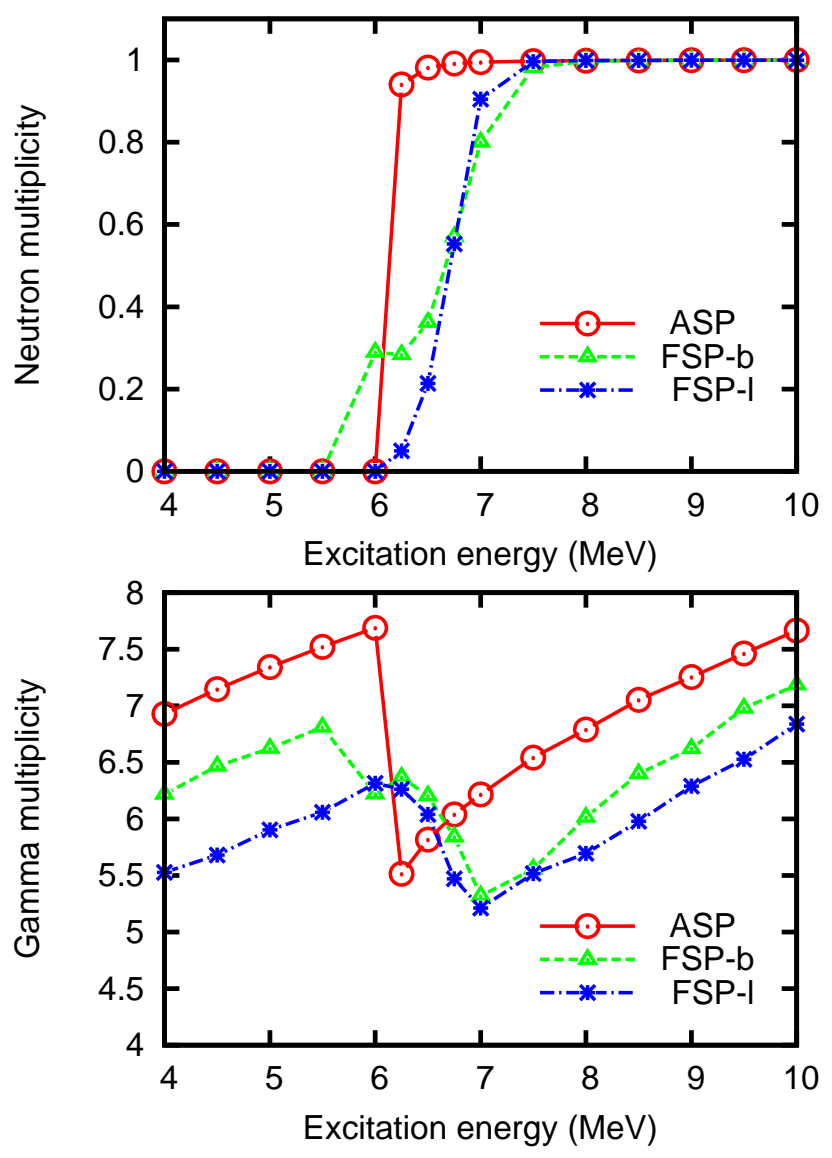

Figure 8: Neutron (top) and gamma (bottom) multiplicities from the deexcitation of ${ }^{143} \mathrm{Ba}$ from a $23 / 2+$ initial state

Concerning the neutron emission, the multiplicity is null up to $5.5 \mathrm{MeV}$. Above that energy, a competition between the neutron and the gamma emission takes place roughly up to 7.5 $\mathrm{MeV}$. The three algorithms are in good agreement in the low and high energy tails but differ strongly in the the neutron/gamma competition zone. The ASP method favors the neutron emission and has a nearly discontinuous behavior at the energy $E_{\text {cut-off }}\left({ }^{142} \mathrm{Ba}\right)$. This behavior is interpreted as a consequence of the numerical issue highlighted in section 2.3. On the other hand, the FSP-1 algorithm predicts a smooth behavior and a much broader neutron/gamma competition zone. The energy of the transition between 0 and 1 neutron emission is no more linked to the artificial rupture at $E_{\text {cut-off }}\left({ }^{142} \mathrm{Ba}\right)$.

Concerning the gamma multiplicity, significant discrepancies between the ASP and FSP methods are noticeable in the whole energy range. Even at low energy where no neutron is emitted, the gamma multiplicity computed with the FSP methods is at least one unit lower than the ASP prediction. In correlation to that, the gamma spectra produced by a FSP method should be harder than expected from a standard ASP technique. To confirm this, we calculate the average energy of the gamma-rays in the same way than the multiplicities. The FSP-l method yields an average gamma-rays energy higher than the ASP method. The difference between the two methods varies from $50 \mathrm{keV}$ up to $500 \mathrm{keV}$ depending on the initial energy. These results show the great sensitivity of the gamma observables to the numerical scheme adopted.

One advantage of the FSP method is that it also provides the uncertainty $\sigma(Q)$ coming from the lack of knowledge on the nuclear structure properties. We present the uncertainties associated with our multiplicity calculation in Fig.9. These quantities are estimated from a Monte Carlo process. Our calculation is therefore accurate up to some statistical uncertainty. No estimator of the statistical uncertainty on $\sigma(Q)$ has been implemented. To check the convergence of our results, we reproduced the Monte Carlo simulation three times with different seeds for the random number generator. The results obtained from these calculations differ roughly by $10 \%$ but lead to the same conclusions.

Concerning the neutron multiplicity, the uncertainty coming from the lack of knowledge in the structure properties is only significant in the neutron/gamma competition zone. In that zone, it is peaked around $0.35 \mathrm{n} /$ cascade. The nuclear structure is not known well enough to lead to an accurate prediction of the neutron emission in that particular energy, spin and parity configuration. For the gamma multiplicity, the structure properties play an important role in the whole energy range. The uncertainty is less peaked and has a significant value outside the neutron/gamma competition zone. In both neutron and gamma cases, the FSP-1 algorithm yields a lower uncertainty than the FSP-b. This is clearly coming from the additional structure information that is injected in the FSP-l method. As shown in Fig. 1, the FSP-l calculation takes into account additional data on 31 levels on ${ }^{143} \mathrm{Ba}$ and a few levels on ${ }^{142} \mathrm{Ba}$. In the case of the gamma multiplicity, the uncertainty is then reduced by a factor two.

\subsubsection{Comparison based on an improved level density}

As discussed in section 3, we expect that important differences between ASP and FSP methods arise when low values of $\rho\left(E_{f}, J_{f}^{\pi}\right) \cdot d E$ are involved in the deexcitation scheme. This situation may happen in a broad range of energy and spin as soon as the bin width $d E$ is small enough. However, we expect it to be particularly critical for final levels close to the Yrast line of the daughter nucleus. On the other hand, the previous comparisons rely on the spin distribution given by Eq. 7 . 


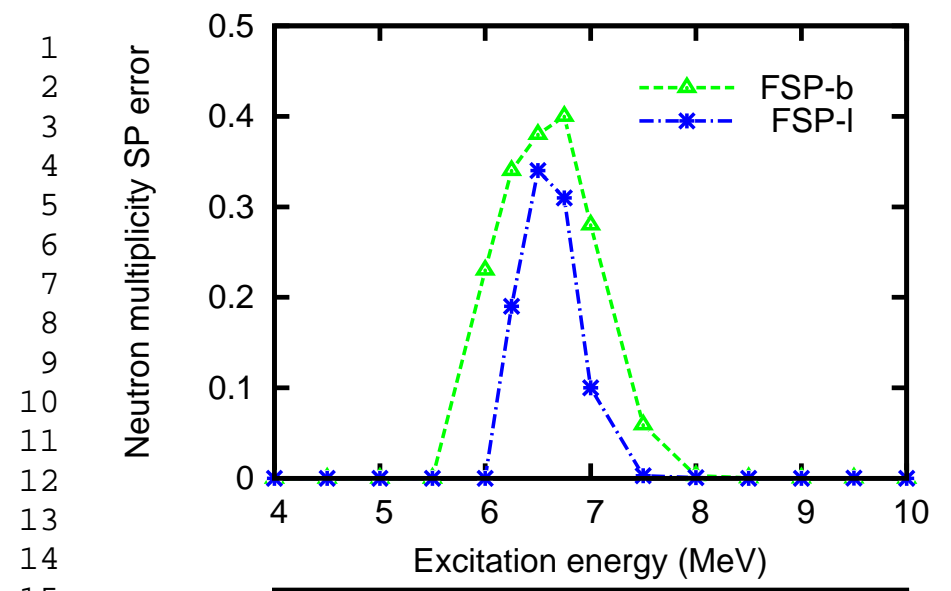

16

17 spin distribution ensures that the level density is null for energies below the Yrast line. To perform our model comparisons, we use a simple analytical estimation of the Yrast line:

$$
\operatorname{Yrast}(E, J)=\frac{J(J+1) \hbar^{2}}{2 I}
$$

The moment of inertia $\mathcal{I}$ is calculated as half of a rigid body moment of inertia.

$$
\mathcal{I}=\frac{1}{2} \mathcal{I}_{\text {rigid }}=\frac{1}{5} A m r^{2}\left(1+0.31 \beta_{2}\right)
$$

with:

$$
r=r_{0} A^{1 / 3}, \quad r_{0}=1.2 \mathrm{fm}, \quad m c^{2}=939.56 \mathrm{MeV}
$$

We inject in this formula the $\beta_{2}$ deformations coming from the AMEDEE database [34]. In the case of the ${ }^{142} \mathrm{Ba}$ the Yrast line starts from 0 at $J=0$ and reaches roughly $2 \mathrm{MeV}$ at $J=10$.
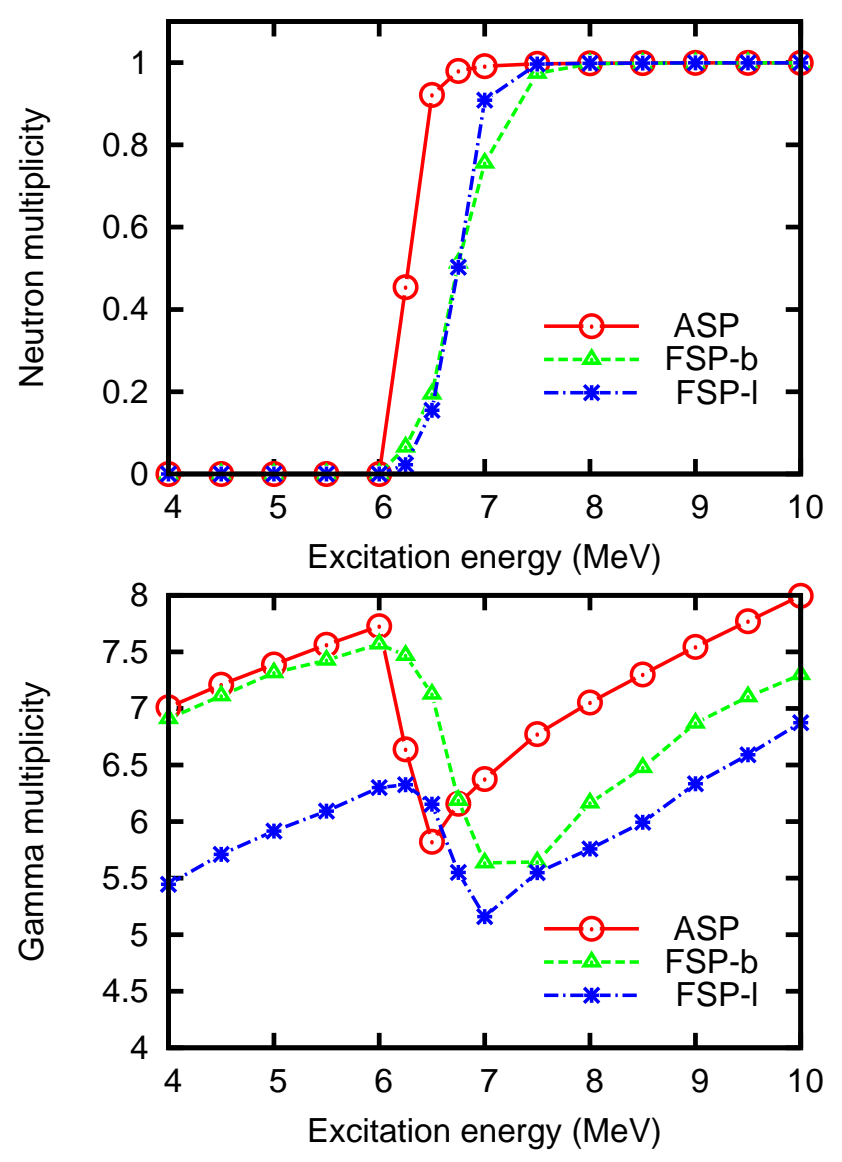

Figure 10: Neutron (top) and gamma (bottom) multiplicities from the deexcitation of ${ }^{143} \mathrm{Ba}$ from a $23 / 2+$ initial state. This calculations rely on the spin distribution described by Eq. 22 that prohibits the existence of levels with energy below the Yrast line

We perform the same deexcitation calculation as in 4.2.1 using the new spin distribution of Eq. 22. Figure 10 presents the neutron and gamma multiplicities obtained. These results still emphasize a great dispersion between the three models. The neutron multiplicity presents the same global feature as in 
Fig. 8. The ASP model overestimates the neutron multiplicity in the transition zone [6.0 MeV; 7.0 MeV] compared to the FSP approaches. This significant difference is the consequence of the numerical issue detailed in section 2. It is still present, even when taking into account explicitly the Yrast line into the level density.

On the other hand, the ASP/FSP-b discrepancy is lower in the present calculations than in the results of section 4.2.1. For instance, the ASP/FSP-b difference of neutron multiplicity at $6.25 \mathrm{MeV}$ decreases from 0.64 without explicit treatment of the Yrast line to 0.39 in this section. Also, for energies below the neutron channel opening, the gamma multiplicity of these two approaches are nearly the same. This is consistent with the fact that Eq. 22 indirectly removes a set of unphysical transitions with low values of $\rho\left(E_{f}, J_{f}^{\pi}\right) \cdot d E$. Moroever, introducing an Yrast line in the level density lowers the variance of the possible nuclear realizations in FSP-b. We therefore expect the FSP-b and ASP methods to give closer results. Finally, we observe that the FSP-1 method is quite insensitive to the addition of the Yrast into the level density. In the FSP-1 approach, the Yrast is already mostly accounted for by the presence of additional experimental levels above $E_{\text {cut-off }}$. These levels must also explain why FSP-1 predicts a gamma multiplicity smaller than ASP/FSP-b from $4 \mathrm{MeV}$ to $6 \mathrm{MeV}$.

\section{Conclusion}

We have highlighted a numerical limitation of the so called "Hauser-Feschbach" method in the framework of deexcitation calculations. This feature is important when calculating neutron/gamma emission cascades starting from a high spin excited state. To circumvent this issue, a different approach called the Fluctuating Structure Properties (FSP) method has been presented. It is based on the idea of sampling the level scheme and transition probabilities within the isotopes present in the deexcitation cascade and takes into account correlations that are often neglected in deexcitation codes. Additionally, it also estimates the uncertainty coming from the lack of knowledge in the structure of the nuclei involved. Two FSP algorithms have been derived and benchmarked on the calculation of the total radiative width for a thermal neutron capture on ${ }^{238} \mathrm{U}$. We compared the standard method with these algorithms for the deexcitation of a ${ }^{143} \mathrm{Ba}$ nucleus in a $23 / 2+$ state. The neutron and gamma multiplicities emphasize significant differences. In particular, the gamma multiplicity predicted by the FSP methods can be 1.5 gamma/cascade lower than the one obtained from the standard "Hauser-Feschbach" algorithm. Most of this discrepancy between the different algorithms holds even when one try to explicitly take into account the Yrast line into the level density formula. Finally, we have shown that the FSP-1 methods offers a convenient way to take into account some incomplete information available in RIPL-3. Injecting this data in our calculation of the gamma multiplicity reduces by a factor of two the uncertainty associated with the lack of knowledge in the structure properties.

\section{Aknowledgement}

This work was partly performed under the auspices of the U.S. Department of Energy by Lawrence Livermore National Laboratory under Contract DE-AC52-07NA27344.

[1] G. Rusev, M. Jandel, M. Krtika, T. A. Bredeweg, A. Couture, T. N. Taddeucci, J. L. Ullmann, Gamma-ray cascade transitions in $\mathrm{Cd}^{112}$ and $\mathrm{Cd}^{114}$ following resonance capture of epithermal neutrons, Phys. Rev. C 87 (5) (2013) 054603. doi:10.1103/PhysRevC.87.054603.

URL http://link.aps.org/doi/10.1103/PhysRevC.87.054603

[2] A. M. Hurst, N. C. Summers, B. W. Sleaford, R. B. Firestone, T. Belgya, Z. S. Revay, Gamma Spectrum from Neutron Capture on Tungsten Isotopes, J. Korean Phys. Soc. 59 (2) (2011) 1491-1494. doi: 10.3938/jkps.59.1491.

[3] F. Corvi, M. Przytula, Spin assignment of neutron resonances via (n, gamma) and (n, alpha) reactions, Phys. Part. Nuclei 35 (6) (2004) 767796.

[4] F. Gunsing, F. Corvi, H. Postma, F. Becvar, The simulation of gamma spectra of $\mathrm{Cd}^{113}(\mathrm{n}, \mathrm{g})$ for neutron resonance spin assignment, Nuclear Instrument and Methods in Physics Research A 365 (1995) 410-418.

URL http://www.sciencedirect.com/science/article/pii/ 016890029500386X

[5] F. Gunsing, E. Berthoumieux, A. Borella, T. Belgya, L. Szentmiklosi, P. Schillebeeckx, J. C. Drohe, R. Wynants, N. Colonna, S. Marrone, G. Tagliente, R. Terlizzi, C. Domingo-Pardo, J. Tain, T. Martinez, C. Massimi, P. M. Mastinu, P. M. Milazzo, Neutron Capture on $\mathrm{Bi}^{209}$ : Determination of the Production Ratio of $\mathrm{Bi}^{210 \mathrm{~m}} / \mathrm{Bi}^{210 \mathrm{~g}}$, J. Korean Phys. Soc. 59 (2) (2011) 1670-1675. doi:10.3938/jkps.59.1670.

[6] I. Stetcu, P. Talou, T. Kawano, M. Jandel, Properties of prompt-fission rays, Phys. Rev. C 90 (2) (2014) 024617. doi:10.1103/PhysRevC. 90. 024617 .

[7] D. Regnier, O. Litaize, O. Serot, A Monte Carlo Simulation of Prompt Gamma Emission from Fission Fragments, EPJ web conf. 42 (2013) 04003. doi:10.1051/epjconf/20134204003.

[8] J. Randrup, R. Vogt, Refined treatment of angular momentum in the event-by-event fission model freya, Phys. Rev. C 89 (4) (2014) 044601. doi:10.1103/PhysRevC.89.044601.

[9] J. R. Huizenga, R. Vandenbosch, Interpretation of Isomeric Cross-Section Ratios for (n,gamma) and (gamma,n) Reactions, Phys. Rev. 120 (4) (1960) 1305-1312. doi:10.1103/PhysRev.120.1305.

[10] J. B. Wilhelmy, E. Cheifetz, R. C. Jared, S. G. Thompson, H. R. Bowman, J. O. Rasmussen, Angular Momentum of Primary Products Formed in the Spontaneous Fission of ${ }^{252}$ Cf, Phys. Rev. C 5 (6) (1972) 2041-2060. doi:10.1103/PhysRevC.5.2041.

[11] K. Skarsvag, Differential angular distribution of prompt gamma rays from spontaneous fission of ${ }^{252}$ Cf, Phys. Rev. C 22 (2) (1980) 638-650. doi : 10.1103/PhysRevC. 22.638. URL http://link.aps.org/doi/10.1103/PhysRevC.22.638

[12] T. Datta, S. P. Dange, A. G. C. Nair, S. Prakash, M. V. Ramaniah, Fission fragment angular momentum: Ratios of independent yields of isomers of ${ }^{95} \mathrm{Nb}$ and ${ }^{132} \mathrm{I}$ in thermal-neutron-induced fission of ${ }^{233} \mathrm{U}$, Phys. Rev. C 25 (1) (1982) 358-364. doi : 10.1103/PhysRevC.25.358. URL http://link.aps.org/doi/10.1103/PhysRevC.25.358

[13] H. Naik, S. P. Dange, R. J. Singh, Angular momentum of fission fragments in low energy fission of actinides, Phys. Rev. C 71 (1) (2005) 014304. doi:10.1103/PhysRevC.71.014304. URL http://link.aps.org/doi/10.1103/PhysRevC.71.014304

[14] S. Mukhopadhyay, L. S. Danu, D. C. Biswas, A. Goswami, P. N. Prashanth, L. A. Kinage, A. Chatterjee, R. K. Choudhury, Prompt spectroscopic studies of fragment nuclei in thermal neutron induced fission of ${ }^{235}$ U, Phys. Rev. C 85 (6) (2012) 064321. doi:10.1103/PhysRevC. 85. 064321.

URL http://link.aps.org/doi/10.1103/PhysRevC.85.064321

[15] G. Boutoux, Neutron-induced cross sections via the surrogate method, Ph.D. thesis, University of Bordeaux I (2011).

[16] W. Hauser, H. Feshbach, The Inelastic Scattering of Neutrons, Phys. Rev. 87 (2) (1952) 366-373. doi : 10.1103/PhysRev . 87. 366. URL http://link .aps .org/doi/10.1103/PhysRev.87.366

[17] F. Becvar, Simulation of gamma cascades in complex nuclei with emphasis on assessment of uncertainties of cascade-related quantities, 
Nucl. Instrum. Methods Phys. Res., Sect. A 417 (2-3) (1998) 434-449. doi:10.1016/S0168-9002(98)00787-6.

URL http://www.sciencedirect.com/science/article/pii/ S0168900298007876

[18] A. C. Wahl, Nuclear-charge distribution and delayed-neutron yields for thermal-neutron-induced fission of ${ }^{235} \mathrm{U},{ }^{233} \mathrm{U}$, and ${ }^{239} \mathrm{Pu}$ and for spontaneous fission of ${ }^{252} \mathrm{Cf}$, Atomic Data and Nuclear Data Tables 39 (1) (1988) 1-156. doi:10.1016/0092-640X (88) 90016-2.

URL http://www.sciencedirect.com/science/article/pii/ $0092640 \times 88900162$

[19] J. Kopecky, M. Uhl, Test of gamma-ray strength functions in nuclear reaction model calculations, Phys. Rev. C 41 (5) (1990) 1941-1955. doi:10.1103/PhysRevC.41.1941. URL http://link.aps .org/doi/10.1103/PhysRevC.41.1941

[20] S. G. Kadmenskij, V. P. Markushev, V. I. Furman, Radiative widths of neutron resonances and giant dipole resonances, Yadernaya Fizika 37 (2) (1983) 277-283.

URL http://inis.iaea.org/Search/search.aspx?orig_q=RN : 15032309

[21] V. A. Plujko, S. N. Ezhov, M. O. Kavatsyuk, A. A. Grebenyuk, R. V. Yermolenko, Testing and improvements of gamma-ray strength functions for nuclear model calculations, in: Proceedings of the international conference on nuclear data for science and technology, Tsukuba, Ibaraki, Japan, 2002.

URL http://inis.iaea.org/Search/search.aspx?orig_q=RN : 34030109

[22] S. Goriely, E. Khan, Large-scale QRPA calculation of E1-strength and its impact on the neutron capture cross section, Nuclear Physics A 706 (12) (2002) 217-232. doi : 10.1016/S0375-9474 (02) 00860-6.

URL http://www.sciencedirect.com/science/article/pii/ S0375947402008606

[23] O. Litaize, O. Serot, Investigation of phenomenological models for the Monte Carlo simulation of the prompt fission neutron and gamma emission, Phys. Rev. C 82 (5) (2010) 054616. doi: 10.1103/PhysRevC. 82. 054616.

URL http://link. aps.org/doi/10.1103/PhysRevC. 82.054616

[24] D. Regnier, O. Litaize, O. Serot, Preliminary Results of a Full Hauser-feshbach Simulation of the Prompt Neutron and Gamma Emission from Fission Fragments, Phys. Proc. 47 (2013) 47-52. doi:10.1016/j.phpro.2013.06.008.

URL http://www.sciencedirect.com/science/article/pii/ S1875389213004379

[25] R. Capote, M. Herman, P. Oblozinsky, P. Young, S. Goriely, T. Belgya, A. Ignatyuk, A. Koning, S. Hilaire, V. Plujko, M. Avrigeanu, O. Bersillon, M. Chadwick, T. Fukahori, Z. Ge, Y. Han, S. Kailas, J. Kopecky, V. Maslov, G. Reffo, M. Sin, E. Soukhovitskii, P. Talou, RIPL - Reference Input Parameter Library for Calculation of Nuclear Reactions and Nuclear Data Evaluations, Nucl. Data Sheets 110 (12) (2009) 3107-3214. doi:10.1016/j.nds . 2009.10.004. URL http://www.sciencedirect.com/science/article/pii/ S0090375209000994

[26] A. Gilbert, A. G. W. Cameron, A Composite Nuclear-Level Density formula with shell corrections, Can. J. Phys. 43 (8) (1965) 1446-1496. doi:10.1139/p65-139.

[27] J. M. Blatt, V. F. Weisskopf, Theoretical nuclear Physics, John Wiley and Sons, 1952

[28] A. J. Koning, S. Hilaire, S. Goriely, Talys 1.4 User Manual (2011). URL http://www.talys. eu/home/

[29] J. R. Grover, J. Gilat, De-Excitation of Highly Excited Nuclei, Phys. Rev. 157 (4) (1967) 802-813. doi:10.1103/PhysRev.157.802. URL http://link. aps .org/doi/10.1103/PhysRev.157.802

[30] J. R. Grover, J. Gilat, Emission of Alpha Particles from Nuclei Having Large Angular Momenta, Phys. Rev. 157 (4) (1967) 823-831. doi:10. 1103/PhysRev. 157.823.

URL http://link.aps.org/doi/10.1103/PhysRev.157.823

[31] J. R. Grover, J. Gilat, Dissipation of Energy and Angular Momentum by Emission of Neutrons and Gamma Rays, Phys. Rev. 157 (4) (1967) 814823. doi:10.1103/PhysRev.157.814.

URL http://link.aps.org/doi/10.1103/PhysRev.157.814

[32] C. E. Porter, R. G. Thomas, Fluctuations of Nuclear Reaction Widths, Phys. Rev. 104 (2) (1956) 483-491. doi : 10.1103/PhysRev. 104. 483.
URL http://link. aps.org/doi/10.1103/PhysRev.104.483

[33] S. F. Mughabghab, Atlas of Neutron Resonances: Resonance Parameters and Thermal Cross Sections. Z=1-100, Elsevier, 2006.

[34] S. Hilaire, M. Girod, The AMEDEE Nuclear Structure Database, AIP Conference Proceedings 1012 (1) (2008) 359. 